\section{Une protéine uNick en son genre}

Mélissa Labelle-Côté, Louise Larose

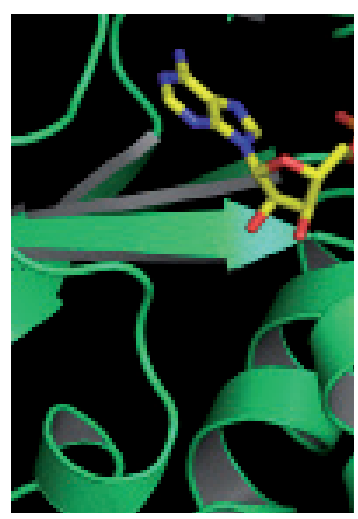

Laboratoire des polypeptides, Faculté de médecine, Université McGill, édifice Strathcona, 3640 , rue University, W315, Montréal, Québec, H3A 2B2 Canada. mélissa.labelle-cote@ umontreal.ca

louise.larose@mcgill.ca

kinase) est unique car non seulement elle possède un domaine $\mathrm{SH}$, mais, de plus, elle présente trois domaines SH3 fonctionnellement différents qui lui confèrent un caractère polyvalent pour assembler une grande diversité de complexes moléculaires. Cette synthèse a pour but de souligner le caractère unique de Nck en faisant état des complexes moléculaires qu'il assemble et des modes particuliers de signalisation qu'il utilise, et en insistant sur sa participation à la progression du cancer.

\section{L'adaptateur Nck} ronnement tient à la transmission d'informations du milieu extracellulaire vers le noyau. Cette opération repose sur un réseau de communication qui intègre différentes voies de signalisation étroitement régulées. Les adaptateurs protéiques, molécules dépourvues d'activité enzymatique et composées uniquement de domaines chargés de lier d'autres protéines, constituent d'importantes composantes de ces processus. Certes, ces protéines unissent physiquement des effecteurs intracellulaires à leurs activateurs, mais, de plus, elles échafaudent différents complexes moléculaires, en influencent la localisation et la stabilité selon le contexte cellulaire, contribuant ainsi à la diversité et à la spécificité de la transmission du signal.

La famille des adaptateurs regroupe notamment des protéines composées exclusivement de domaines homologues de Src (protein tyrosine kinase homolog of the Rous sarcoma virus) de type 2 (SH2) et de type 3 (SH3). Sauf exception, les domaines SH2 lient des protéines phosphorylées sur des résidus tyrosine ( $p y)$, tandis que les domaines $\mathrm{SH} 3$ s'associent à des protéines contenant une région riche en résidus proline. Parmi ces adaptateurs, la protéine Nck (non catalytic region of tyrosine
Chez les mammifères, Nck est représenté par Nckl (Nck $\alpha$ ) et Nck2 (Nck $\beta$, Grb4) [1]. Nckl a été découvert lors du criblage d'une banque d'expression d'ADNc de mélanome humain [2]. Nck2 a été mis en évidence chez la souris à partir d'une banque d'expression d'ADNc embryonnaire [3]. Ces deux protéines sont identiques à $69 \%$ tant chez l'homme que chez la souris. Leurs domaines SH partagent jusqu'à $85 \%$ d'identité. On note que les régions entre ces domaines sont peu conservées (Figure 1) [1, 4]. Des études génétiques chez la souris ont révélé que les gènes Nck sont essentiels au développement embryonnaire et fonctionnellement redondants [5]. Cette redondance pourrait s'expliquer parce que de nombreux partenaires d'interaction sont communs aux deux Nck (Figure 1). Néanmoins, on leur attribue aussi des fonctions uniques comme le suggèrent leur interaction avec des partenaires différents, leur expression simultanée dans certains types cellulaires et leurs profils d'expression tissulaire distincts $[1,5]$.

Le mode classique de signalisation signal/py-SH2/SH3-effecteurs utilisé en général par les adaptateurs contenant des domaines $\mathrm{SH}$ est le mécanisme principal par lequel Nck propage l'information aux effecteurs intracellulaires suivant l'activation de récepteurs membranaires de type tyrosine kinase (RTK : receptor tyrosine kinases) (Figure 2) [1]. Cependant, Nck transmet aussi le signal selon un mode de signalisation inversé où l'information perçue par les domaines SH3 est transmise à 


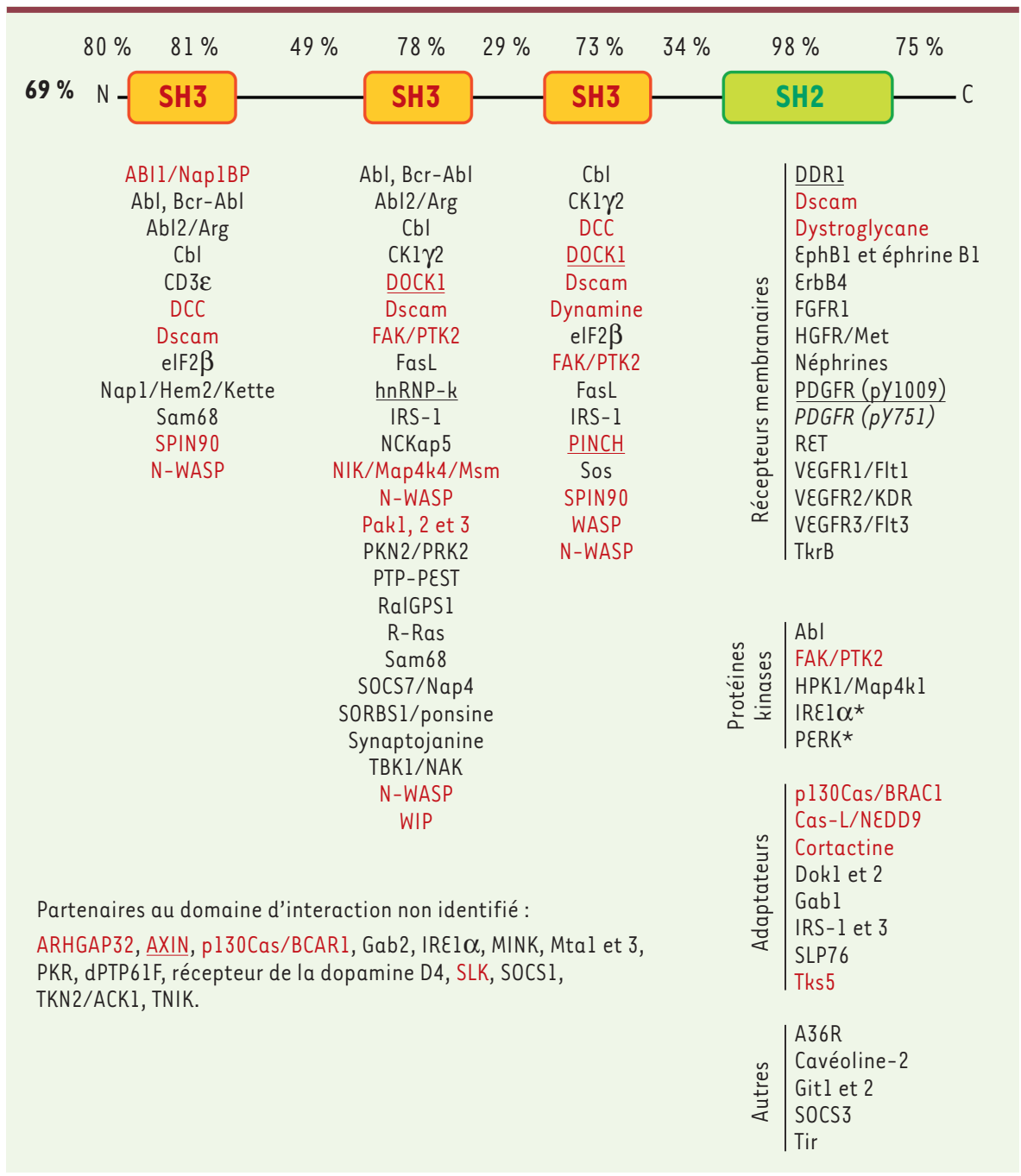

Figure 1. Nck et ses partenaires d'interaction. Représentation schématique de la structure des adaptateurs Nckl et Nck2 et pourcentage d'identité entre les différentes régions de Nckl (NP_006144.1) et Nck2 (NP_001004720.1) déterminés par LALIGN (www.ch.embnet.org). Protéines associées aux domaines SH : spécifiques à Nckl (italique); spécifiques à Nck2 (soulignées); impliquées dans la régulation de l'organisation du cytosquelette d'actine (rouge) et *non publiées [L. Larose]. Adapté de [30] et complété selon les informations disponibles dans la base de données EntrezGene [http://ncbi.nlm.nih. gov/gene].

un effecteur qui se lie au domaine SH2 (Figure 2) $[6,7]$. En plus, Nck interagit par son domaine $\mathrm{SH} 2$ ou ses domaines $\mathrm{SH} 3$ avec des protéines transmembranaires à la surface du réticulum endoplasmique (RE) pour moduler la signalisation à partir du RE (Figure 2) [8-10]. Finalement, en utilisant ses domaines $\mathrm{SH} 3$, Nck se lie à des protéines dans le noyau $[11,12]$, suggérant que cet adaptateur pourrait participer aussi à des processus de signalisation dans ce compartiment cellulaire (Figure 2). La distribution subcellulaire de Nck concorde avec son rôle d'adaptateur dans différents compartiments cellulaires. Nck est présent de façon diffuse dans le cytoplasme et le noyau. En revanche, il s'accumule dans certaines régions de la membrane plasmique et au niveau du RE. Cette distribution est régie par son interaction constitutive (par ses SH3) ou dynamique (par son SH2) avec d'autres protéi- nes et régule la transmission du signal. Nck étant la cible de plusieurs protéines kinases, sa phosphorylation pourrait également influencer son interaction avec certains partenaires. D'autre part, Nck présente des régions riches en résidus proline pouvant servir de site de liaison pour des protéines régulatrices contenant un domaine SH3. Notamment, Nck2 présente entre ses $1^{\text {er }}$ et $2^{e}$ domaines $\mathrm{SH} 3$ une séquence d'acides aminés ([K/R] $X[K / R] R X X S$ ) capable d'interagir

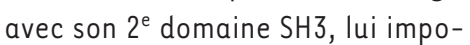
sant ainsi une conformation inhibitrice qui masque ce domaine de liaison [13]. Une telle interaction intramoléculaire suggère l'existence d'un mécanisme de sélection autorisant la liaison du $2^{\mathrm{e}}$ domaine $\mathrm{SH} 3$ uniquement par des effecteurs de haute affinité.

\section{Les multiples fonctions de Nck}

\section{La polymérisation de l'actine}

La majorité des processus biologiques associés à Nck engagent la régulation du cytosquelette d'actine. Que ce soit au niveau des podocytes des cellules épithéliales rénales, de la synapse immune formée par les lymphocytes $T$, de la migration des axones neuronaux, de l'entrée de pathogènes dans la cellule ou de la régulation de la motilité cellulaire, Nck sert de pont entre des récepteurs membranaires et les protéines qui régissent la polymérisation de l'actine. Dans cette perspective, Nck favorise la polymérisation de l'actine en participant directement à l'échafaudage de complexes moléculaires contenant WASP (Wiskott-Aldrich syndrome proteins) ou WAVE1 (WASP family verprolin-homologous protein) qui facilitent l'activation du complexe protéique Arp2/3 (actin-related protein) déterminant l'amorce et l'élongation des embranchements du cytosquelette d'actine [14]. Nck participe aussi indirectement à l'activation de ces complexes en orchestrant l'activation des petites protéines GTPasiques de la famille de Rho (Cdc42, Racl et RhoA). Pour ce faire, il recrute des protéines activatrices de ces 


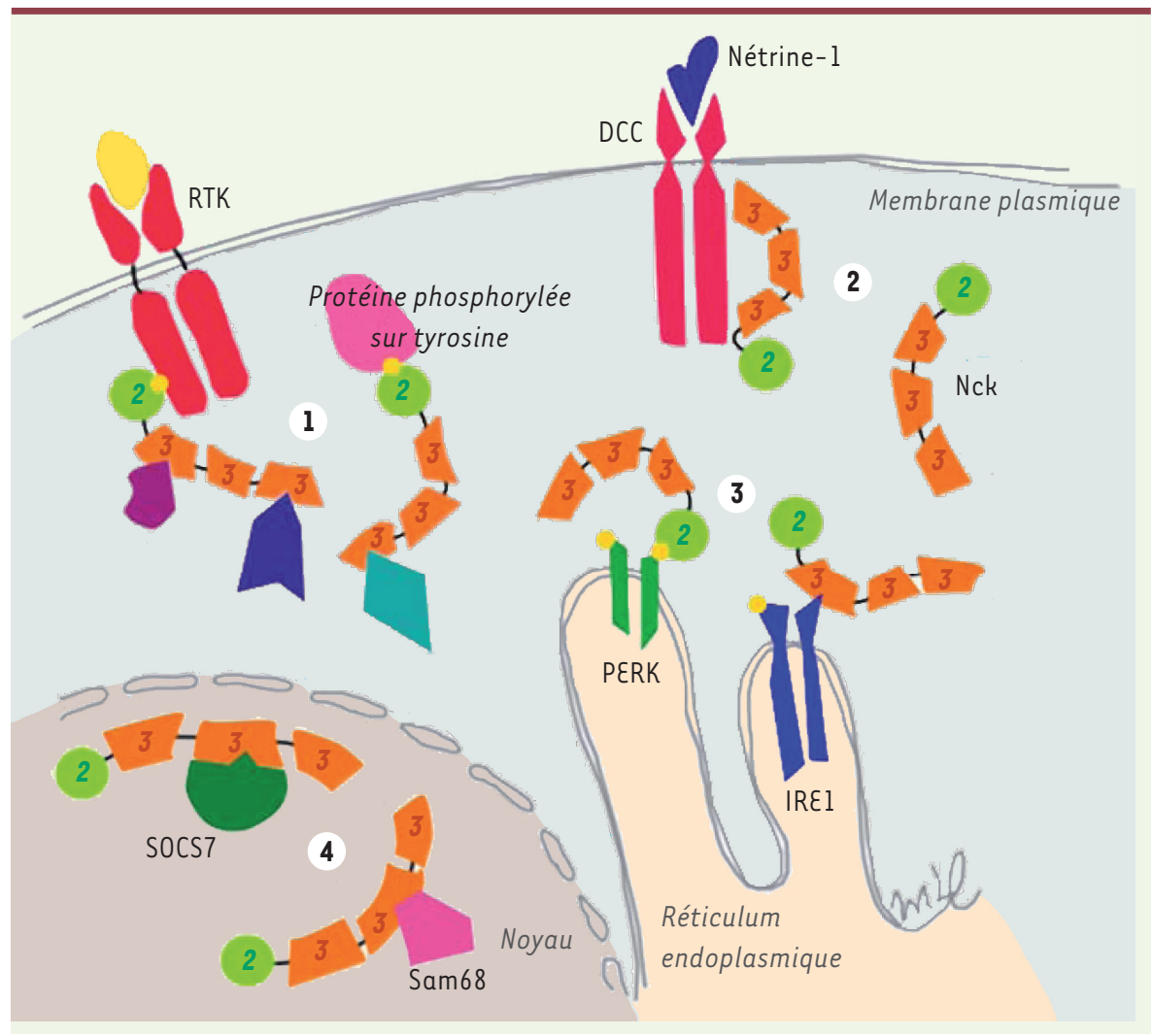

Figure 2. Modes de signalisation par Nck. Suivant le mode de signalisation traditionnel des adaptateurs à domaines $\mathrm{SH} 2$ et $\mathrm{SH} 3$ (signal/py-SH2/SH3-effecteurs), Nck participe à la transmission du signal par des récepteurs membranaires à activité tyrosine kinase ou des protéines cytosoliques phosphorylées sur tyrosine (1). Nck adopte un mode de transmission du signal inversé (signal-SH3/SH2-py/effecteurs) pour transmettre le signal à partir de certains récepteurs membranaires auxquels il est lié par ses domaines $\mathrm{SH} 3$ (2). Nck lie aussi des protéines transmembranaires du réticulum endoplasmique par son domaine SH2 ou ses domaines SH3 (3). Nck est transféré au noyau lorsqu'il s'associe par ses domaines SH3 à des protéines localisées au noyau (4) (๔ Mélissa Labelle-Côté, 2010).

GTPases (GEF : guanine nucleotide exchange factor) comme PIX (PAKinteracting exchange factor) et DOCK1 (dedicator of cytokinesis 1), tout en associant simultanément des effecteurs de ces dernières tel PAK (p21-activated protein kinase). En rapprochant physiquement tous ces acteurs, Nck contribue à la signalisation intracellulaire dirigée vers la réorganisation du cytosquelette d'actine.

\section{L'adhésion, la migration et l'invasion cellulaires}

Les intégrines régulent l'organisation du cytosquelette d'actine dans les complexes d'adhérence responsables de l'interaction des cellules avec la matrice extracellulaire. Le complexe protéique intégrinestaline-paxilline-FAK (focal adhesion kinase)-Src constitue la base de la signalisation des intégrines dirigée vers le cytosquelette d'actine $[15,31]$. Nck participe à ce complexe signalétique en associant directement FAK (Figure 3) [15]. Cependant, plusieurs observations confèrent un rôle spécifique à Nck2 dans la signalisation des intégrines. D'abord, contrairement à Nck1, Nck2 lie la GEF DOCK1 qui permet l'activation de Racl nécessaire à la réorganisation de l'actine par les intégrines [16]. Ensuite, Nck2, par l'intermédiaire de son association à PINCH (particularly interesting cys-his-rich protein) dans le complexe moléculaire PINCH-ILK (integrin-like kinase), participe à la signalisation des intégrines régissant l'étalement, la migration et la survie cellulaires. La liaison à PINCH, tout comme celle à DOCKI, sans faire appel à une région riche en résidus proline, dépend tout de même du $3^{e}$ domaine $\mathrm{SH} 3$ de Nck2 [17] : ceci démontre encore une fois le caractère atypique de cet adaptateur. En étant recruté au domaine cytosolique des intégrines par le complexe moléculaire ILK-PINCH ou la kinase FAK et en s'associant simultanément aux récepteurs à activité tyrosine kinase, Nck assure la convergence de la signalisation amorcée par ces deux types de récepteurs vers une réponse cellulaire harmonieuse.

Nck participe aussi à l'adhésion entre les cellules au niveau des jonctions adhérentes. II contribue indirectement à la formation et à la stabilité de ces contacts intercellulaires érigés essentiellement par les $\varepsilon$-cadhérines en liant la cortactine au sein du complexe moléculaire cortactine-WIP (WASP-interacting protein)-WASP-Arp2/3 responsable de la polymérisation de l'actine dans ces structures (Figure 3) [18, 19]. L'importance de Nck pour les jonctions adhérentes a d'abord été illustrée dans le rein. En s'associant au récepteur membranaire néphrine par son domaine $\mathrm{SH} 2$ et en recrutant par ses domaines $\mathrm{SH} 3$ la machinerie qui régit la polymérisation de l'actine, Nck est au cœur de la formation des jonctions adhérentes qui assurent le maintien de l'intégrité de la fente de filtration entre les pédicelles des podocytes indispensable à la fonction rénale [20].

Nck joue un rôle important dans la migration cellulaire comme l'a démontré un défaut de migration et d'organisation de l'actine dans les lamellipodes des fibroblastes murins embryonnaires dépourvus de l'activité des deux gènes Nck [5]. Par l'échafaudage des complexes moléculaires DOCK1-Racl-WAVE-Arp2/3 (Figure 3) et Cdc42-WASP-Arp2/3, Nck participe respectivement à la 

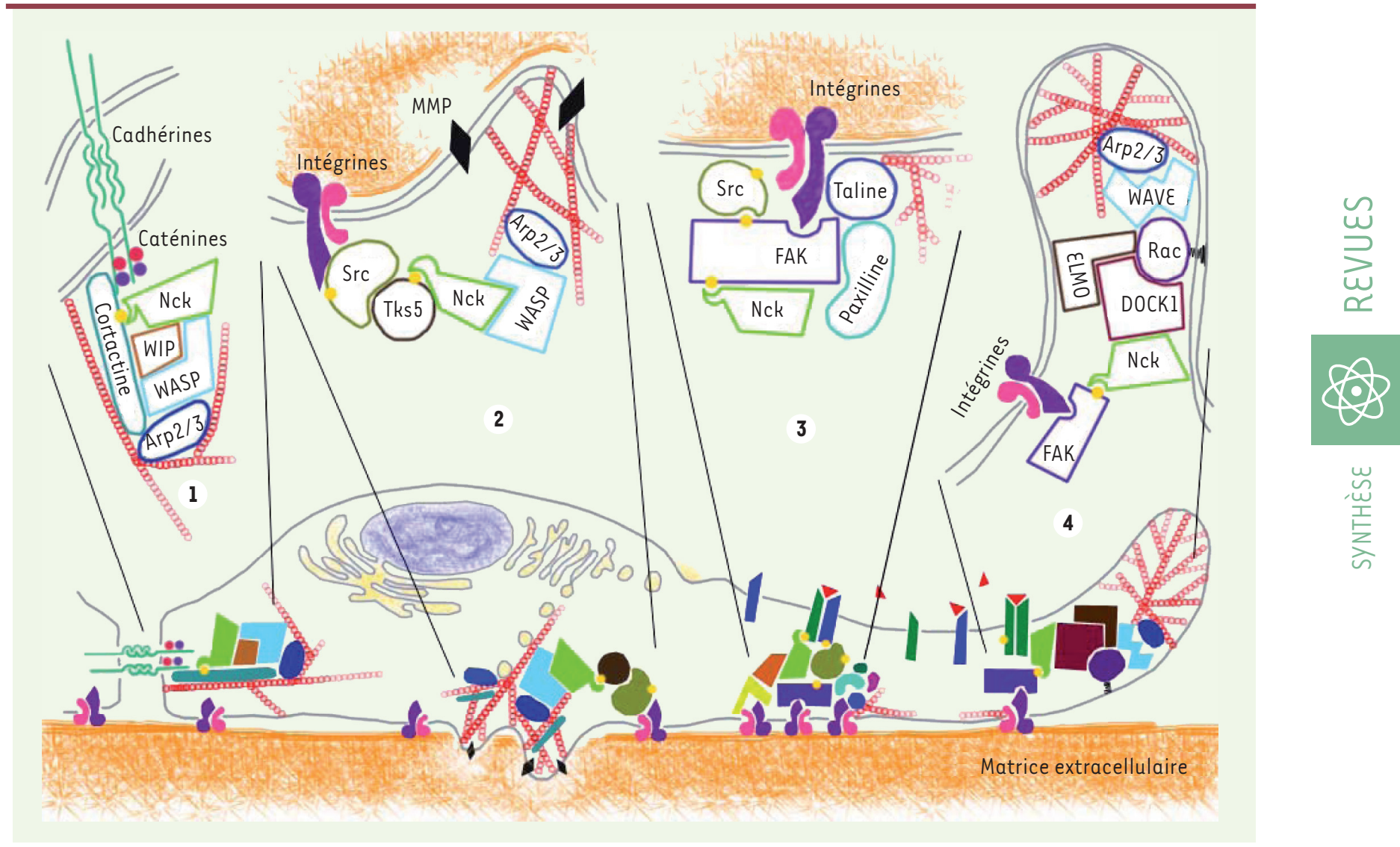

Figure 3. Nck et le cytosquelette d'actine. Représentation schématique de certains des complexes moléculaires associés à Nck et importants dans la régulation du cytosquelette d'actine. 1. Jonctions adhérentes : après l'activation des $\varepsilon$-cadhérines dans un contact intercellulaire, la protéine kinase Src phosphoryle la cortactine qui lie les $\varepsilon$-cadhérines et y enrôle la machinerie de polymérisation de l'actine. Nck se lie à la cortactine phosphorylée sur tyrosine et interagit aussi avec WIP et WASP. 2. Invadopodes : après l'adhésion des intégrines à la matrice extracellulaire, la kinase Src phosphoryle Tks5 sur tyrosine. Nck se lie à Tks 5 phosphorylée sur tyrosine et y enrôle la machinerie de polymérisation de l'actine, incluant WASP, Arp2/3 et la cortactine. La présence de métalloprotéases (MMP) sécrétées ou liées à la membrane permet la dégradation de la matrice extracellulaire et facilite l'invasion. 3. Complexes d'adhérence : l'engagement des intégrines avec la matrice extracellulaire active et recrute la protéine kinase FAK au complexe d'adhérence. Nck en se liant à FAK est recruté dans ce complexe. Les complexes moléculaires ILK-PINCH-Nck2 et paxilline-PKL-PIX-PAK-Nck non représentés participent aussi à la signalisation des intégrines. 4. Lamellipodes : la polymérisation de l'actine nécessaire à la formation des lamellipodes est régulée par la GTPase Racl. La GEF DOCKl et l'adaptateur ELMO contribuent à l'activation de Racl. Nck2, en s'associant à FAK et à DOCK1, permet de diriger le complexe moléculaire DOCK1-ELMO à proximité de Racl au point d'ancrage des intégrines (๔ Mélissa Labelle-Côté, 2010).

formation des lamellipodes qui poussent le front de migration de la cellule et à celle des filopodes qui explorent l'espace extracellulaire [21]. D’autre part, une étude récente a révélé que Nckl se localise spécifiquement dans les invadopodes, tandis que les podosomes sont caractérisés par la présence de la protéine Grb2 (growth factor receptor-bound protein 2), autre adaptateur à domaines $\mathrm{SH}$ [22]. Les podosomes et les invadopodes sont des structures de la membrane plasmique riches en actine et formées à la face ventrale des cellules. Par l'intermédiaire des intégrines, ces structures servent de domaines d'adhésion, mais elles sont surtout caractérisées par la présence de métalloprotéases dégradant la matrice extracellulaire. Contrairement aux podosomes, la formation d'invadopodes est restreinte aux cellu- les cancéreuses auxquelles ils confèrent un potentiel invasif. Dans les invadopodes, Nck est lié à Tks5 et à la cortactine, ainsi son rôle d'adaptateur est essentiel dans les complexes moléculaires intégrines-Src-Tks 5 et cortactine-WIP-WASP-Arp2/3 pour stimuler la polymérisation de l'actine responsable de la formation et de l'activité invasive des invadopodes (Figure 3) [23].

\section{La traduction et la réponse au stress}

Nck influence l'initiation de la traduction par son interaction directe avec la sous-unité $\beta$ du facteur d'initiation elF2 [24]. II favorise l'activité d'elF2 en 
atténuant la phosphorylation de sa sous-unité $\alpha$ par certaines protéines kinases activées en réponse à différents stress cellulaires [25]. Par exemple, Nck module la phosphorylation d'elF2 par PERK (PKR-related endoplasmic reticulum kinase), protéine kinase de la membrane du RE activée en réponse à un stress causé par l'accumulation de protéines mal conformées dans le $R E$, en recrutant dans un même complexe elF2 et la phosphatase PPlc [9]. Nck module aussi la signalisation d'IREl $\alpha$ (inositol requiring enzyme 1 ), autre protéine de la membrane du RE qui possède une activité kinase et une activité endonucléase activées dans les mêmes conditions de stress [10, 32]. L'importance de Nck dans ce contexte s'est récemment accrue avec la découverte que les souris dont le gène $\mathrm{Nckl}$ est inactivé sont protégées de la résistance à l'insuline résultant de l'activation anormale de la signalisation par IREl $\alpha$ induite par l'obésité [26]. D'autre part, Nck joue aussi un rôle dans la réponse des cellules aux rayons UV, stress génomique qui provoque le transfert de Nck au noyau après son interaction avec SOCS7 (suppressor of cytokine signaling) [11]. Cette étude a démontré que Nck est requis pour l'arrêt du cycle cellulaire provoqué par les UV et qu'une baisse importante des niveaux cytosoliques de Nck résultant de sa redistribution au noyau pourrait indirectement causer la réorganisation du cytosquelette d'actine observée en réponse aux UV.

\section{Nck et le processus de transformation oncogénique}

L'attribution à Nckl d'un potentiel oncogénique provient des observations selon lesquelles sa surexpression dans des fibroblastes murins induit leur transformation, et la formation par ces derniers de tumeurs lorsqu'ils sont injectés chez la souris [27]. D'autre part, la découverte fortuite de Nckl à partir de mélanome humain [2] laissait présager un taux élevé d'ARNm dans ce type de cancer. Cette hypothèse, confirmée par une analyse de micropuces d'ADN [28], est maintenant fortement étayée par les résultats de plusieurs études (répertoriés dans la base de données transcriptomiques 0 ncomine ${ }^{1}$ ) qui démontrent que l'expression de Nckl ou Nck2 est significativement augmentée dans de nombreuses lignées de cellules cancéreuses humaines. En revanche, même s'il apparaît maintenant que le caractère oncogénique des deux Nck fait appel à leur capacité de potentialiser la transformation cellulaire induite par des oncogènes forts [4], le mécanisme par lequel cet adaptateur collabore au développement tumoral demeure encore inconnu.

Nck pourrait contribuer au développement initial de cancers en participant à la signalisation activée par de nombreux récepteurs de facteurs de croissance : récepteurs de l'EGF (epidermal growth factor), du PDGF (platelet-derived growth factor) et de I'HGF (hepatocyte growth factor), dont les activités dérégulées sont étroitement associées à une perte de la maîtrise de la prolifération cellulaire dans plusieurs cancers. De plus, en modulant la signalisation de PERK et d'IREl $\alpha$, Nck pourrait faciliter l'adaptation et la survie des cellules tumorales aux conditions de stress créées par les faibles teneurs en oxygène et en nutriments qui prévalent lors de la croissance de tumeurs. D'autre part, son rôle d'adaptateur dans la signalisation du récepteur du VEGF

${ }^{1}$ https://www.oncomine.org. (vascular endothelial growth factor) pourrait aussi lui conférer une grande importance dans les processus angiogéniques contribuant à la progression tumorale. La motilité des cellules cancéreuses se caractérise essentiellement par deux types de mouvements migratoires : amiboïdiens, adoptés par des cellules arrondies présentant de nombreuses déformations globulaires de la membrane plasmique, et mésenchymateux, utilisés par des cellules allongées qui présentent des lamellipodes et possèdent une activité protéolytique dégradant la matrice extracellulaire. Le passage d'un mode de migration à l'autre permet aux cellules de s'adapter à leur environnement et les GTPases Rho exercent une influence déterminante sur ce processus. En effet, si RhoA est associée aux mouvements amiboïdiens et Racl au mode de type mésenchymateux, Cdc42 participe pour sa part aux deux modes selon les effecteurs qu'elle associe [29]. Nck est dynamiquement présent dans l'univers des GTPases Rho. Entre autres, il lie des protéines de la famille de Cas (Crk-associated substrates) en amont de Racl et la protéine kinase PAK en aval de Racl, ainsi que PAK et WASP en aval de $\mathrm{Cdc} 42$. II est donc plausible qu'une variation des niveaux de Nck dans les cellules cancéreuses puisse influencer la sélection du mode de migration adopté et contribuer ainsi à la progression du cancer. D'autre part, Nck est recruté par les protéines Tks 5 et cortactine, il est ainsi activement concentré dans les invadopodes, structures d'invasion exclusives aux cellules cancéreuses. Durant le développement de la tumeur, l'important transfert de Nck dans les invadopodes pourrait alors se faire au détriment des complexes d'adhérence et des jonctions adhérentes, facilitant ainsi le détachement des cellules cancéreuses de la tumeur primaire.

En résumé, Nck est une composante importante des processus de transformation oncogénique par son rôle dans l'orchestration de la prolifération cellulaire, de la réponse des cellules aux conditions de stress et des processus régissant la migration cellulaire. De plus, durant la progression du cancer, il est possible que Nck, intensément mobilisé dans certaines structures cellulaires, y assemble de nouveaux complexes moléculaires favorisant la migration et l'invasion au détriment de l'adhésion dépendante des contacts cellule-cellule ou cellule-matrice extracellulaire (Figure 3).

\section{Conclusion et perspectives}

Nck, adaptateur à domaines homologues de Src, est sollicité par des voies de signalisation qui régissent une gamme importante de fonctions cellulaires. Nos 


\section{GLOSSAIRE}

Complexes d'adhérence : larges complexes protéiques dynamiques constituant le site d'ancrage du cytosquelette d'actine à la matrice extracellulaire ; parfois abrégés FA (focal adhesion).

Filopodes : longues et fines projections membranaires qui explorent l'espace extracellulaire à la recherche d'informations; composés de fibres d'actine parallèles dont la polymérisation est principalement induite par la GTPase Cdc42.

Invadopodes : projections membranaires apparentées aux podosomes, mais propres aux cellules cancéreuses invasives; souvent associés à une intense activité protéolytique dégradant la matrice extracellulaire. Jonctions adhérentes : lieu de contact entre deux cellules voisines; stabilisées par l'interaction des cadhérines à la surface des deux cellules et par l'association du cytosquelette d'actine du côté intracellulaire. Lamellipodes : larges structures membranaires qui dirigent le front de migration de la cellule; composés d'un réseau de filaments d'actine entrecroisés dont la polymérisation est induite par la GTPase Racl.

Podosomes : structures membranaires cylindriques composées de filaments d'actine perpendiculaires au plan de la cellule servant de microdomaine d'adhérence au niveau de la portion ventrale de la cellule. connaissances des protéines Nck montrent l'existence de nouveaux modes de signalisation pour ce type d'adaptateurs et leur contribution aux processus de signalisation amorcés dans des compartiments intracellulaires autres que la membrane plasmique. De nombreuses études devront approfondir la contribution des protéines adaptatrices comme Nck à la progression du cancer, ce qui aiderait aussi au développement de nouveaux outils thérapeutiques ayant ces protéines comme cibles principales pour le traitement et la prévention du cancer. Dans cette optique, l'accent devrait être mis sur les mécanismes qui régissent la formation et les fonctions des invadopodes, incluant le rôle de Nck dans ces structures. Le rôle de Nck dans la réponse $d u R \varepsilon$ au stress mérite aussi une attention particulière vu l'importance grandissante de ce processus dans le développement de différentes pathologies telles le cancer et le diabète de type 2 . Une étude plus systémique du rôle des protéines Nckl et Nck2 dans les processus biologiques, incluant les processus oncogéniques, permettrait également de mieux définir les subtilités qui les distinguent. Finalement, nos connaissances des fonctions de l'adaptateur Nck augmentent notre compréhension de l'univers de la signalisation cellulaire, mais en révèlent aussi toute la complexité. $\diamond$

\section{SUMMARY}

\section{A uNick protein}

$\mathrm{Nck}$ is an adaptor protein composed of three $\mathrm{N}$-terminal Src Homology (SH) 3 domains followed by a unique C-terminal SH2 domain. Like other $\mathrm{SH} 2 / \mathrm{SH} 3$ domains-containing adaptor proteins, Nck mediates signal transduction from activated cell surface receptors by directing the flow of information to elicit properly orchestrated cell responses. In this way, Nck appears to be unique in its contribution to a wide variety of cellular processes. Moreover, in addition to the typical signal/py-SH2/SH3-effectors mode of signaling, Nck also trans- duces signals through an inverse mode of signaling (signal-SH3/SH2-py/effectors) and from various cell compartments. Since Nck contributes to important morphogenic and mitogenic processes, deregulated expression of Nck could be detrimental to cellular homeostasis. In agreement, Nck expression has been found upregulated in numerous types of cancer. In this paper we delineate the main molecular signaling complexes associated with Nck, focusing on those involved in cancer progression. $\diamond$

\section{CONFLIT D'INTÉRÊTS}

Les auteurs déclarent n'avoir aucun conflit d'intérêts concernant les données publiées dans cet article.

\section{REMERCIEMENTS}

Étant donné le grand nombre d'études publiées ayant pour sujet Nck, nous nous excusons si certaines références pertinentes n'ont pas été citées faute d'espace. Louise Larose est chercheur national du FRSP (Fonds de recherche en santé du Québec).

\section{RÉFÉRENCES}

1. Chen M, She H, Davis EM, et al. Identification of Nck family genes, chromosomal localization, expression, and signaling specificity. J Biol Chem $1998 ; 273: 25171-8$.

2. Lehmann JM, Riethmuller G, Johnson JP. Nck, a melanoma cDNA encoding a cytoplasmic protein consisting of the src homology units $\mathrm{SH} 2$ and $\mathrm{SH} 3$. Nucleic Acids Res 1990 ; 18 : 1048.

3. Margolis B, Silvennoinen O, Comoglio F, et al. High-efficiency expression/ cloning of epidermal growth factor-receptor-binding. Proc Natl Acad Sci USA $1992 ; 89: 8894-8$.

4. Braverman LE, Quilliam LA. Identification of Grb4/Nckbeta, a src homology 2 and 3 domain-containing adapter protein having similar binding and biological properties to Nck. J Biol Chem 1999 ; 274 : 5542-9.

5. Bladt F, Aippersbach $\varepsilon$, Gelkop S, et al. The murine Nck SH2/SH3 adaptors are important for the development of mesoderm-derived embryonic structures and for regulating the cellular actin network. Mol Cell Biol 2003 ; $23:$ 4586-97.

6. Li X, Meriane M, Triki I, et al. The adaptor protein Nck-1 couples the netrin-1 receptor DCC (deleted in colorectal cancer) to the activation of the small GTPase Racl through an atypical mechanism. J Biol Chem $2002 ; 277$ : 37788-97.

7. Rao Y, Zipursky SL. Domain requirements for the Dock adapter protein in growth- cone signaling. Proc Natl Acad Sci USA 1998 ; 95 : 2077-82.

8. Kebache $S$, Cardin $\varepsilon$, Nguyen DT, et al. Nck-1 antagonizes the endoplasmic reticulum stress-induced inhibition of translation. J Biol Chem 2004 ; 279 : 9662-71.

9. Latreille M, Larose L. Nck in a complex containing the catalytic subunit of protein phosphatase 1 regulates eukaryotic initiation factor 2 alpha signaling and cell survival to endoplasmic reticulum stress. J Biol Chem 2006 ; $281: 26633-44$.

10. Nguyen DT, Kebache $S$, Fazel A, et al. Nck-dependent activation of extracellular signal-regulated kinase- 1 and regulation of cell survival during endoplasmic reticulum stress. Mol Biol Cell 2004 ; 15 : 4248-60.

11. Kremer BE, Adang LA, Macara IG. Septins regulate actin organization and cell-cycle arrest through nuclear accumulation of NCK mediated by SOCS7. Cell $2007 ; 130: 837-50$.

12. Lawe $D C$, Hahn C, Wong AJ. The Nck SH2/SH3 adaptor protein is present in the nucleus and associates with the nuclear protein SAM68. Oncogene 1997 ; $14: 223-31$.

13. Takeuchi K, Sun ZY, Park S, Wagner G. Autoinhibitory interaction in the multidomain adaptor protein Nck: possible roles in improving specificity and functional diversity. Biochemistry $2010 ; 49$ : 5634-41 


\section{RÉFÉRENCES}

14. Takenawa T, Suetsugu S. The WASP-WAVE protein network: connecting the membrane to the cytoskeleton. Nat Rev Mol Cell Biol $2007 ; 8: 37-48$.

15. Cornillon J, Campos L, Guyotat D. Focal adhesion kinase (FAK), une protéine aux fonctions multiples. Med Sci (Paris) $2003 ; 19: 743-52$.

16. Tu Y, Kucik DF, Wu C. Identification and kinetic analysis of the interaction between Nck-2 and DOCK180. FEBS Lett $2001 ; 491: 193-9$.

17. Tu Y, Li F, Wu C. Nck-2, a novel Src homology2/3-containing adaptor protein that interacts with the LIM-only protein PINCH and components of growth factor receptor kinase-signaling pathways. Mol Biol Cell $1998 ; 9$ : 3367-82.

18. Ren $G$, Helwani FM, Verma $S$, et al. Cortactin is a functional target of $\varepsilon$-cadherin-activated Src family kinases in MCF7 epithelial monolayers. J Biol Chem 2009 ; 284 : 18913-22.

19. Tehrani S, Tomasevic N, Weed S, et al. Src phosphorylation of cortactin enhances actin assembly. Proc Natl Acad Sci USA 2007 ; 104 : 11933-8.

20. Audard V, Lang $P$, Sahali $D$. Pathogénie du syndrome néphrotique à lésions glomérulaires minimes. Med Sci (Paris) $2008 ; 24: 853-8$.

21. Eden S, Rohatgi R, Podtelejnikov AV, et al. Mechanism of regulation of WAVEl-induced actin nucleation by Racl and Nck. Nature $2002 ; 418: 790-3$.

22. Oser M, Dovas A, Cox D, Condeelis J. Nckl and Grb2 localization patterns can distinguish invadopodia from podosomes. Eur J Cell Biol. 2011 ; 90 : 181-8.

23. Stylli SS, Stacey $\Pi$, Verhagen AM, et al. Nck adaptor proteins link Tks5 to invadopodia actin regulation and ECM degradation. J Cell Sci 2009; $122: 2727-40$.

24. Kebache $S$, Zuo D, Chevet $\varepsilon$, Larose L. Modulation of protein translation by Nck-1. Proc Natl Acad Sci USA 2002 ; 99 : 5406-11.
25. Cardin $\varepsilon$, Latreille M, Khoury C, et al. Nck-1 selectively modulates elF2alphaSer51 phosphorylation by a subset of elF2alpha-kinases. FEBS ) $2007 ; 274: 5865-75$

26. Latreille M, Laberge MK, Bourret $G$, et al. Deletion of Nckl attenuates hepatic $\varepsilon R$ stress signaling, improves glucose. Am J Physiol Endocrinol Metab 2011 ; 300 : ع423-34

27. Chou MM, Fajardo JE, Hanafusa H. The SH2- and SH3-containing Nck protein transforms mammalian fibroblasts in the absence of elevated phosphotyrosine levels. Mol Cell Biol $1992 ; 12$ : 5834-42.

28. De Wit NJ, Rijntjes J, Diepstra JH, et al. Analysis of differential gene expression in human melanocytic tumour lesions by custom made oligonucleotide arrays. BrJ Cancer $2005 ; 92: 2249-61$.

29. Gadea G. Migration des cellules tumorales : GEF et GAP montrent le chemin. Med Sci (Paris) $2009 ; 25: 343-5$.

30. Li W, Fan J, Woodley DT. Nck/Dock: an adapter between cell surface receptors and the actin cytoskeleton. Oncogene $2001 ; 20: 6403-17$

31. Albiges-Rizo C, Bouvard D, Bouin AP, et al. La taline : une allure d'haltérophile et la pratique du stretching pour mieux transmettre les forces. Med Sci (Paris) 2009 ; 25 : 909-11.

32. Bouchecareilh $M$, Chevet $\varepsilon$. Stress du réticulum endoplasmique : une réponse pour éviter le pIRE. Med Sci (Paris) 2009 ; 25 : 281-7.

TIRÉS À PART

M. Labelle-Côté et L. Larose

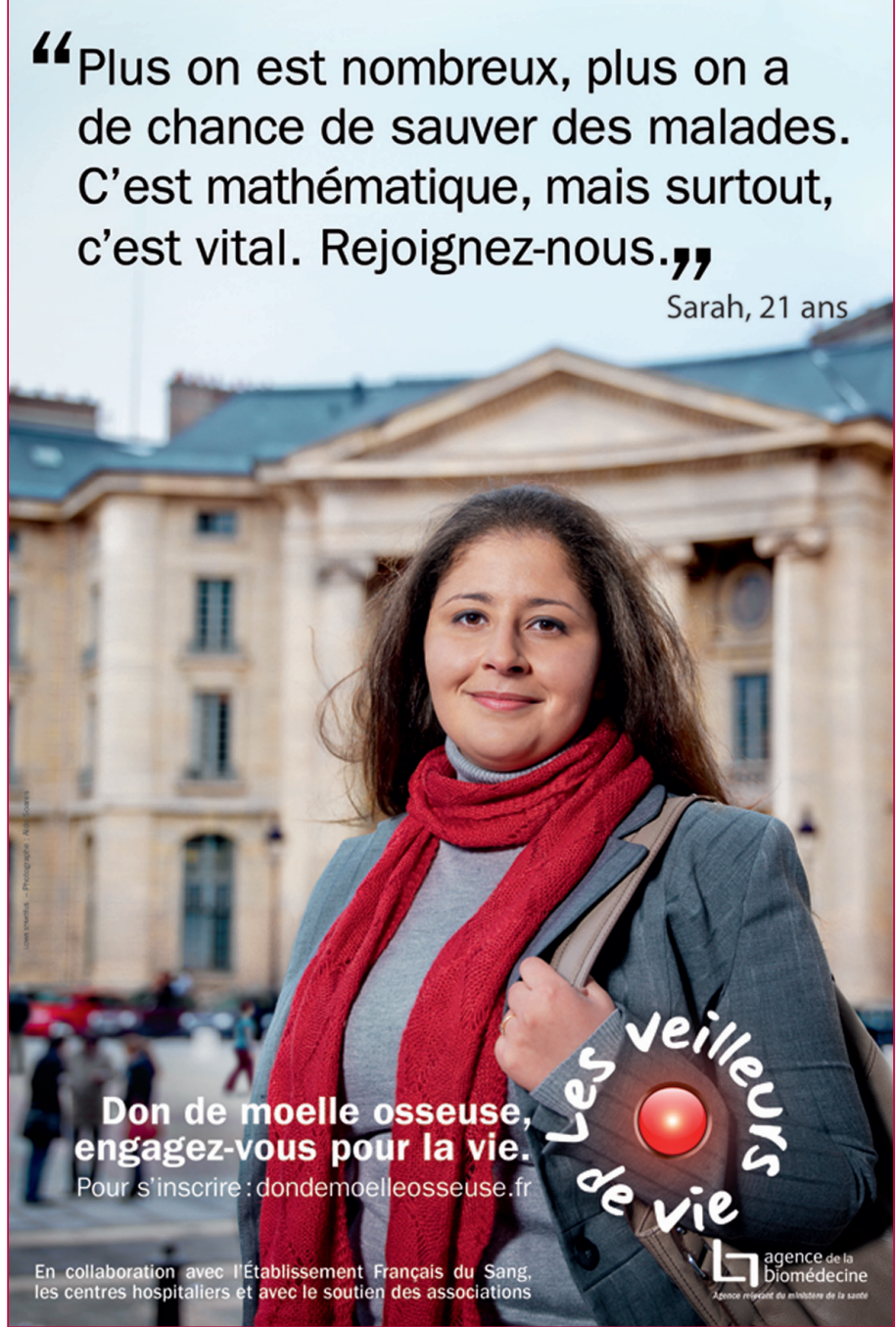

